ARTICLE

Received 30 Jul 2015 | Accepted 19 Jan 2016 | Published 26 Feb 2016

DOl: $10.1038 /$ ncomms10777

OPEN

\title{
Phosphatidylinositol phosphate kinase PIPKI $\gamma$ and phosphatase INPP5E coordinate initiation of ciliogenesis
}

Qingwen $\mathrm{Xu}^{1}$, Yuxia Zhang ${ }^{2}$, Qing Wei ${ }^{2}$, Yan Huang ${ }^{1}$, Jinghua $\mathrm{Hu}^{1,2} \&$ Kun Ling ${ }^{1}$

Defective primary cilia are causative to a wide spectrum of human genetic disorders, termed ciliopathies. Although the regulation of ciliogenesis is intensively studied, how it is initiated remains unclear. Here we show that type I $\gamma$ phosphatidylinositol 4-phosphate (Ptdlns(4)P) 5-kinase (PIPKI $\gamma$ ) and inositol polyphosphate-5-phosphatase E (INPP5E), a Joubert syndrome

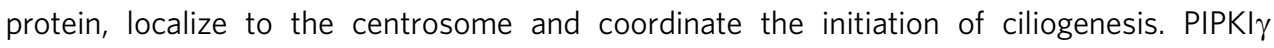
counteracts INPP5E in regulating tau-tubulin kinase-2 (TTBK2) recruitment to the basal body, which promotes the removal of microtubule capping protein CP110 and the subsequent axoneme elongation. Interestingly, INPP5E and its product-Ptdlns(4)P-accumulate at the centrosome/basal body in non-ciliated, but not ciliated, cells. Ptdlns(4)P binding to TTBK2 and the distal appendage protein CEP164 compromises the TTBK2-CEP164 interaction and inhibits the recruitment of TTBK2. Our results reveal that Ptdlns(4)P homoeostasis, coordinated by PIPKI $\gamma$ and INPP5E at the centrosome/ciliary base, is vital for ciliogenesis by regulating the CEP164-dependent recruitment of TTBK2.

\footnotetext{
${ }^{1}$ Department of Biochemistry and Molecular Biology, Mayo Clinic, Rochester, Minnesota 55905, USA. 2 Division of Nephrology and Hypertension, Mayo Clinic, Rochester, Minnesota 55905, USA. Correspondence and requests for materials should be addressed to J.H. (email: hu.jinghua@mayo.edu) or to K.L. (email: ling.kun@mayo.edu).
} 
P rimary cilia, the critical microtubule-based organelles that sense the environmental chemical and/or mechanical cues, regulate the homoeostasis of various organs/tissues in vertebrates $^{1-3}$. While the overall biogenesis of the cilium is known, key aspects remain unclear. Early steps in ciliogenesis include the docking of the mother centriole (M-centriole)/basal body to the plasma membrane ${ }^{4-6}$, removal of the microtubule capping protein CP110 from the distal end of the M-centriole/ basal body that allows the initiation of axoneme nucleation ${ }^{7}$, recruitment of intraflagellar transport (IFT) complexes $^{8-10}$ and formation of a transition zone (TZ) ${ }^{11-13}$, followed by further extension of the microtubule axoneme and the ciliary membrane. Recruitment of TTBK2 to the M-centriole/basal body by the distal appendage/transition fibre (TF) protein CEP164 is essential for the removal of CP110 from the distal end of the M-centriole/basal body and the initiation of axoneme assembly ${ }^{14,15}$. However, the mechanism regulating TTBK2 recruitment remains elusive.

Phosphoinositides (PIs) are generated by phosphorylation of phosphatidylinositol (PtdIns) at the 3, 4 and/or 5 positions of the inositol ring. By the spatially localized recruitment of effector proteins ${ }^{16,17}$, PI species regulate various important membrane/ protein trafficking or cytoskeleton-related cellular processes throughout the cell ${ }^{18,19}$. Protein recruitment often occurs through a specific PI-binding domain on the protein. For instance, pleckstrin homology $(\mathrm{PH})^{20,21}$, Phox homology, Fab1, YOTB, Vac 1, EEA1 (FYVE), epsin N-terminal homologue domains and so on, as well as less-conserved basic motifs, bind to PIs with varying affinities and specificities ${ }^{16,22}$. PI binding usually mediate the targeting of the protein to a specific membrane compartment and/or induce a conformational change that regulates the interaction between the protein and its binding partner ${ }^{16}$. Lately, a few studies implicated PI signalling in the context of cilia and ciliopathies. For example, three PI 5phosphatases localize to cilia and are correlated with ciliopathies (that is, INPP5E in Joubert syndrome and nephronophthisis ${ }^{23,24}$, and OCRL and INPP5B in Lowe syndrome $\left.{ }^{25,26}\right)$. PtdIns $(4,5) \mathrm{P}_{2}$, a substrate of INPP5E, is required for flagella outgrowth during Drosophila spermatogenesis ${ }^{27}$ and reduced in rodent polycystic kidney disease models ${ }^{28,29}$. Recently, two groups independently reported that INPP5E localizes in primary cilia and maintains a PtdIns(4)P-high, PtdIns(4,5) $\mathrm{P}_{2}$-low environment, which ensures the processing of hedgehog signalling by inhibiting the ciliary entry of TULP3 and Gpr161 (refs 30,31). These studies clearly support the importance of PIs in ciliary signalling; however, whether and how these phospholipids participate in ciliogenesis is unclear.

In current study, we show that PIPKI $\gamma$, a centrosomal PtdIns(4)P 5-kinase ${ }^{32}$, presents at the basal body in ciliated cells. INPP5E also resides at the M-centriole in serum-fed, nonciliated cells; however, translocates to cilium proper in ciliated cells. We find that PIPKI $\gamma$ is required for, but INPP5E inhibits, ciliogenesis. Consistently, these two PI enzymes regulate the recruitment of TTBK2 to the $\mathrm{M}$-centriole in an opposite manner. Further investigation demonstrates a centrosomal pool of PtdIns(4)P, a product of INPP5E and the substrate of PIPKI $\gamma$, prevents the recruitment of TTBK2 to the M-centriole by binding to CEP164 and TTBK2, and inhibiting the TTBK2-CEP164 interaction. Overall, these discoveries reveal a novel mechanism that PIPKI $\gamma$ and INPP5E coordinate the initiation of ciliogenesis by spatiotemporally regulating a centrosomal PtdIns(4)P pool to control the TTBK2 recruitment and CP110 removal. a

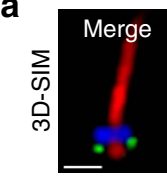

b

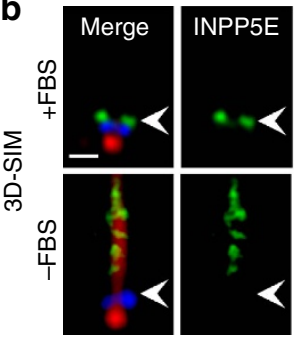

f

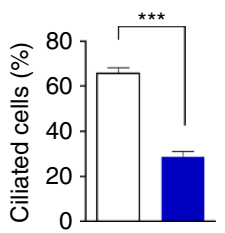

$\square$ siNC

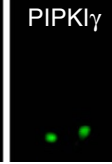

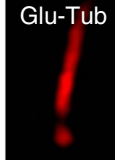
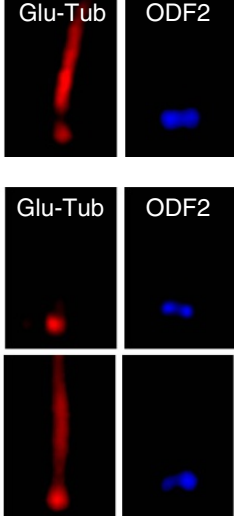

g

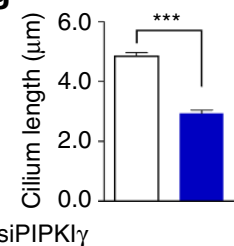

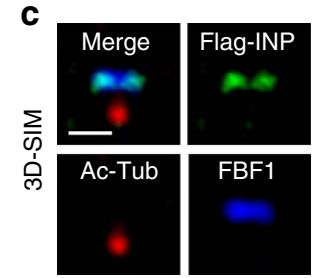
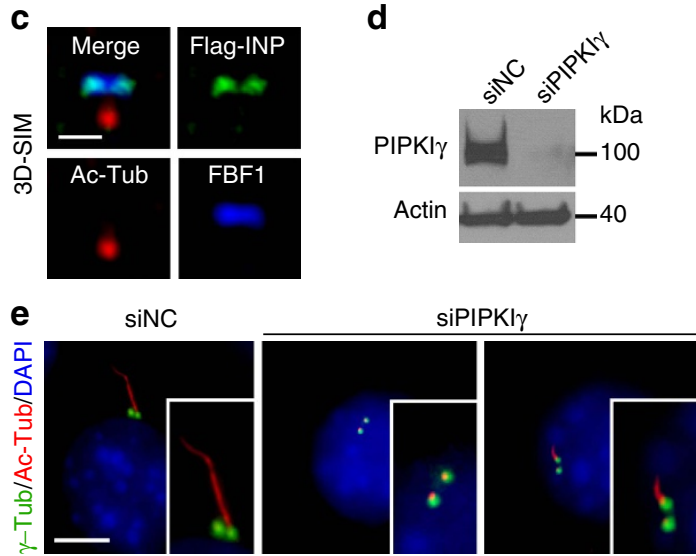

h
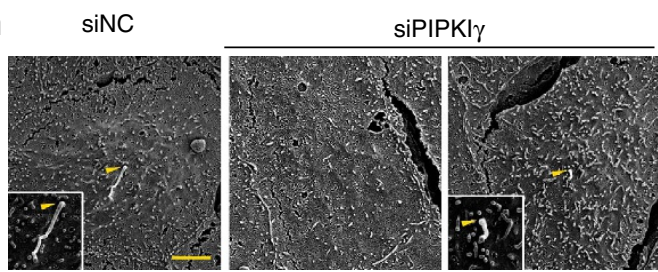

Figure 1 | PIPKI $\gamma$ is required for ciliogenesis. (a-c) RPE-1 cells were treated as described below, subjected to indirect immunofluorescence (IF) labelling with indicated antibodies, and than analysed with three-dimensional structured illumination microscopy (3D-SIM). (a) Serum-starved RPE-1 cells; (b) RPE-1 cells before ( + FBS) and after ( - FBS) 24h serum starvation; (c) RPE-1 cells expressing Flag-tagged INPP5E (Flg-INP).Ac-Tub, acetylated $\alpha$-tubulin; GluTub, polyglutamylated tubulin. (d) IMCD3 cells were treated with negative control (siNC) or PIPKI $\gamma$-specific (siPIPKI $\gamma$ ) siRNAs for 48 h. (e-g) IMCD3 cells described in $\mathbf{d}$ were serum starved for $24 \mathrm{~h}$ and subjected to IF. Nuclei were visualized by DAPI staining (blue). Insets show magnified images of primary cilia. $\gamma$-Tub, $\gamma$-tubulin. Percentage of ciliated cells $(\mathbf{f})$ and cilium length $(\mathbf{g})$ in each group $(n>200)$ were quantified from three independent experiments and plotted. Error bars represent s.d. in $\mathbf{f}$ and s.e.m. in $\mathbf{g}$. ${ }^{\star \star \star} P<0.001$. (h) Cells described in $\mathbf{d}$ were analysed by scanning electron microscopy. Arrowheads indicate the ciliary base. Scale bar, $0.5 \mu \mathrm{m}$ in $\mathbf{a}-\mathbf{c}$; Scale bar, $5 \mu \mathrm{m}$ in $\mathbf{e}$ and $\mathbf{h}$. 


\section{Results}

PIPKI $\gamma$ at the basal body is necessary for ciliogenesis. Our previous work describing the function of PIPKI $\gamma$ at the centrosome $^{32}$ suggests that PIPKI $\gamma$ might also participate in the context of cilia when the M-centriole transforms into the basal body. To test this, we first investigated the subcellular localization of PIPKI $\gamma$ in ciliated cells using a validated anti-PIPKI $\gamma$ antibody $^{32}$. As shown in Fig. 1a, PIPKI $\gamma$ was visualized at the ciliary base in human retinal pigment epithelial (RPE-1) cells, but not in cells treated with PIPKI $\gamma$-specific siRNAs (Supplementary Fig. 1a,b). We further confirmed this basal-body localization of PIPKI $\gamma$ in other types of ciliated cells, including mouse inner medullary collecting duct (IMCD3) cells, NIH3T3 cells and human renal cortical tubular epithelial (RCTE) cells (Supplementary Fig. 1c). Images obtained by super-resolution three-dimensional structured illumination microscopy revealed that PIPKI $\gamma$ localizes below the distal end marker ODF2 in RPE-1 cells (Fig. 1a) and forms a ring-like structure with similar diameter as the centrosome protein PLK4 (Supplementary Fig. 1f). Meanwhile, PIPKI $\alpha$ and PIPKI $\beta$ antibodies yielded no cilium-related signal (Supplementary Fig. 1d,e), indicating that PIPKI $\gamma$ is the sole type I PIP kinase at the basal body.

Recent studies have implicated PI 5-phosphatase INPP5E in the context of cilia $^{23,30,31,33}$; thus, we examined the spatial relationship between INPP5E and PIPKI $\gamma$. Distinct from the proximal localization pattern for PIPKI $\gamma$, INPP5E resides slightly above ODF2 (Fig. 1b, upper panels) and colocalizes with the distal appendage protein FBF1 (Fig. 1c) on the M-centriole in nonciliated cells. In ciliated cells, INPP5E is no longer detected at the basal body (Fig. 1b, lower panels), and presents only in the ciliary lumen as reported ${ }^{23,33}$. Considering PIPKI $\gamma$ and INPP5E are counteracting enzymes, these results suggest that the level of corresponding PIs (PtdIns $(4) \mathrm{P}$ and/or PtdIns $\left.(4,5) \mathrm{P}_{2}\right)$ around the $\mathrm{M}$-centriole/basal body ought to be changed before and after ciliogenesis.

We next sought to determine the physiological importance of PIPKI $\gamma$ at the ciliary base. Using the specific siRNAs ${ }^{32}$, we found that knockdown of PIPKI $\gamma$, but not PIPKI $\alpha$ or PIPKI $\beta$, significantly inhibited ciliogenesis in various types of mammalian cells (Fig. 1d-g; Supplementary Fig. 1g-i). In the small portion of PIPKI $\gamma$-depleted cells that still formed cilia, the average cilium length was about half of that in control cells (Fig. 1e,g). These phenotypes were confirmed by scanning electron microscopy (Fig. 1h). Interestingly, ppk-1 (the solo PIPKI in Caenorhabditis elegans)-knockout nematodes also exhibit defective ciliogenesis (Supplementary Fig. 1j,k), suggesting the function of PIPKI $\gamma$ in ciliogenesis likely evolves early across ciliated species.

Loss of PIPKI $\gamma$ affects TZ but not TF assembly. To understand how PIPKI $\gamma$ regulates ciliogenesis, we carefully examined PIPKI $\gamma$-depleted cells. TFs, which are transformed from the distal appendages of the M-centriole, are important for tethering the basal body to the plasma membrane $e^{4,5}$ and docking the IFT particles to the basal body ${ }^{34}$. In PIPKI $\gamma$-depleted RCTE (Fig. 2a) and RPE-1 (Supplementary Fig. 2) cells, TF components (CEP83, SCLT1, CEP164 and FBF1) maintain their regular localizations at the ciliary base. Consistently, PIPKI $\gamma$-depleted cells displayed normal basal body docking to the plasma membrane (Fig. 2b), which also suggests that TFs are formed properly when PIPKI $\gamma$ is absent. We then inspected the localization of $\mathrm{TZ}$ proteins. Interestingly, although the localization of MKS components MKS1 and TCTN1 was not affected by depletion of PIPKI $\gamma$, no NPHP1 was detected on the basal body (Fig. 2c,d; Supplementary Fig. 2), indicating that NPHP1 recruitment is interrupted. These data suggest that PIPKI $\gamma$ acts after basal body docking and before
TZ formation. Capping protein CP110 suppresses microtubule nucleation; therefore it must be removed from the M-centriole to allow TZ assembly and axoneme elongation when ciliogenesis is induced ${ }^{7}$. Thus, we next examined whether PIPKI $\gamma$ is required for CP110 removal.
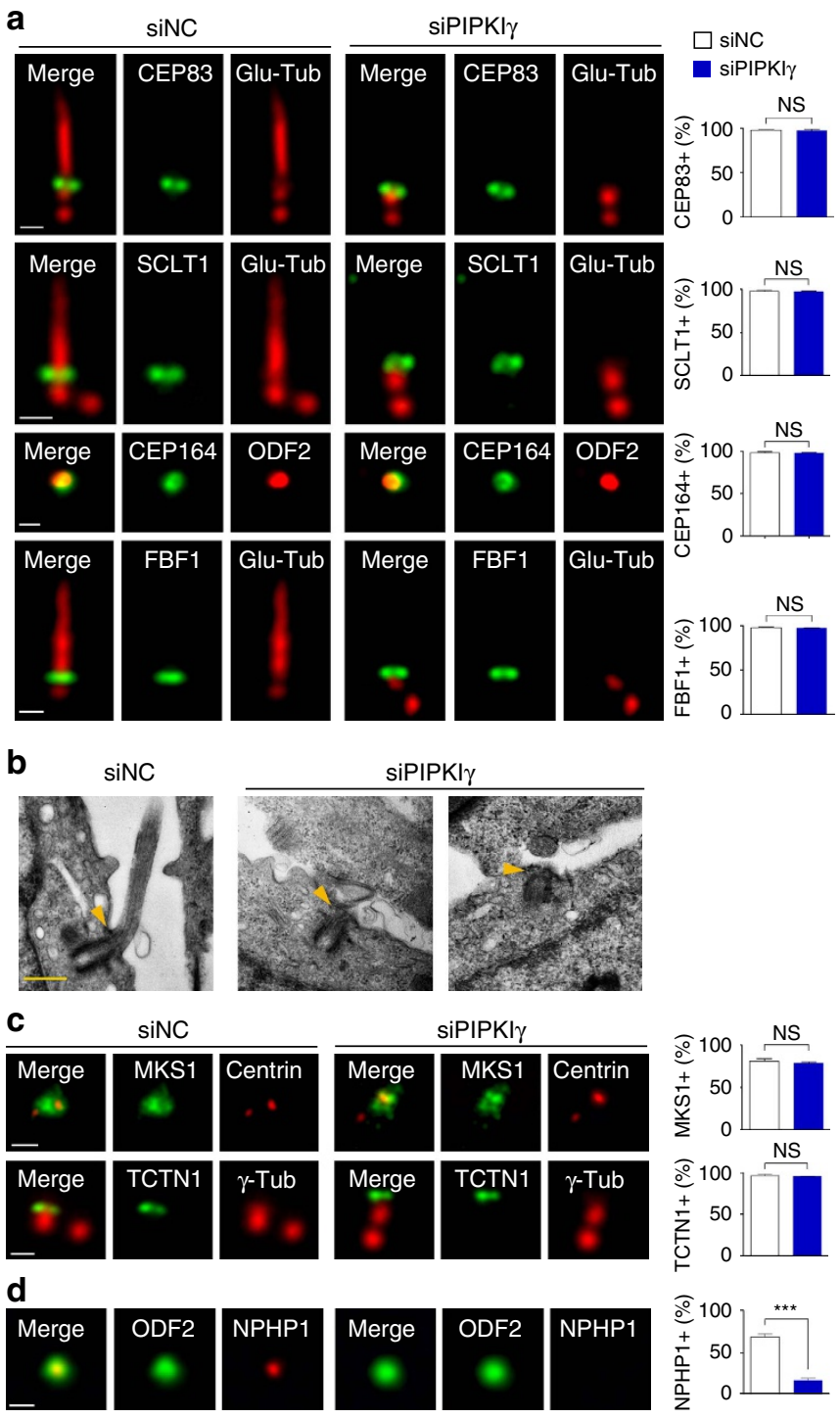

Figure 2 | PIPKI $\gamma$-depleted cells exhibit normal basal body docking to the plasma membrane. (a,c,d) RCTE cells were treated with negative control (siNC) or PIPKI $\gamma$-specific (siPIPKI $\gamma$ ) siRNAs for $48 \mathrm{~h}$ and serum starved for $24 \mathrm{~h}$, then subjected to indirect IF microscopy analyses with indicated antibodies. Glu-Tub, polyglutamylated tubulin; $\gamma$-Tub, $\gamma$-tubulin. Scale bars, $0.5 \mu \mathrm{m}$. (a) The localization of transition fibre proteins is not affected by PIPKI $\gamma$ depletion. (b) The basal body docking to the plasma membrane is not changed by PIPKI $\gamma$ depletion. Control (siNC) or PIPKI $\gamma$-depleted (siPIPKI $\gamma$ ) IMCD3 cells grown on coverslips were serum starved for $48 \mathrm{~h}$ and then fixed, scraped off the coverslips, spun into pellets and processed for transmission electron microscopy. Arrowheads point to distal ends of the basal body. (c) Localization of the components of transition zone MKS module is not affected by PIPKI $\gamma$ depletion. (d) NPHP1 lost its association with the M-centriole/basal body in PIPKI $\gamma$-depleted cells. Percentage of cells $(n>60)$ exhibiting centrosome/basal body localization of tested transition fibre (a) or transition zone $(\mathbf{c}, \mathbf{d})$ proteins in control or PIPKI $\gamma$-depleted cells was quantified from three independent experiments and plotted. Error bars represent s.d. NS, no statistically significant difference. ${ }^{\star \star \star} P<0.001$. 
PIPKI $\gamma$ regulates for TTBK2 recruitment and CP110 removal. Remarkably, in PIPKI $\gamma$-depleted cells, CP110 is abnormally preserved on both centrioles on serum starvation (Fig. 3a; Supplementary Fig. 3a). Depletion of CP110 restores the axoneme elongation in PIPKI $\gamma$-deficient cells (Fig. 3b), suggesting that PIPKI $\gamma$ functions upstream of CP110 during ciliogenesis. It is known that the removal of CP110 depends on the recruitment of TTBK2 to the M-centriole/basal body on serum starvation or growth inhibition ${ }^{14}$. In agreement with the sustained CP110, we found no TTBK2 at the M-centriole/basal body after serum starvation in PIPKI $\gamma$-depleted cells (Fig. 3c; Supplementary Fig. $3 \mathrm{~b}$ ). In the context that the global protein levels of TTBK2 and CP110 were not changed by PIPKI $\gamma$ depletion (Fig. $3 \mathrm{~d}$ ), we propose that PIPKI $\gamma$ is required for the serum starvation-induced translocation of TTBK2 to the M-centriole/basal body, which subsequently promotes the removal of CP110, and then TZ assembly and axoneme elongation.

Since PIPKI $\gamma$ is a lipid kinase, we then asked whether its enzyme activity is required for the recruitment of TTBK2 to the M-centriole. As reported, the C-terminus-truncated PIPKI $\gamma$ (PIPKI $\gamma \Delta \mathrm{CT}$ ) exhibits specific targeting to centrioles with stronger association with the $\mathrm{M}$-centriole ${ }^{32}$, unlike the full-length PIPKI $\gamma$ that also localizes to other subcellular locales such as the plasma membrane, cell adhesions and vesicle compartments $^{35,36}$. Therefore, we utilized PIPKI $\gamma \Delta \mathrm{CT}$ to eliminate phenotypes unrelated to primary cilia. Notably, overexpression of the active PIPKI $\gamma \Delta \mathrm{CT}$, but not the kinasedead mutant (D253/316A), can promote the recruitment of TTBK2 to the M-centriole even without serum starvation (Fig. $3 \mathrm{e}-\mathrm{g}$ ), indicating that excessive PIPKI $\gamma$ kinase activity stimulates the recruitment of TTBK2 to the M-centriole independent of serum starvation. These effects were reproduced in cells overexpressing the full-length wild-type or kinase-dead PIPKI $\gamma$ (Supplementary Fig. 3c). Accompanied with the increased TTBK2 at the centrosome, CP110 signal at the M-centriole was substantially decreased but not entirely vanished in cells ectopically expressing PIPKI $\gamma \Delta \mathrm{CT}$ (Fig. 3h,i), suggesting that the TTBK2 recruited to the $\mathrm{M}$-centriole by overexpressed PIPKI $\gamma \Delta \mathrm{CT}$ is functional but not sufficient to completely remove CP110. Indeed, ectopically expressed PIPKI $\gamma \Delta \mathrm{CT}$ did not induce serum starvation-independent ciliogenesis (Supplementary Fig. 3d).

INPP5E inhibits TTBK2 recruitment and CP110 removal. Our results suggest that the kinase activity of PIPKI $\gamma$ at the $\mathrm{M}$-centriole/basal body is indispensable for the early stage of ciliogenesis. Therefore, we expect that INPP5E, which localizes at the centrosome in serum-fed cells and potentially counteracts PIPKI $\gamma$ on site, would neutralize PIPKI $\gamma$ function in TTBK2 recruitment. Indeed, knockdown of INPP5E led to a substantial increase of TTBK2 signal on the M-centriole in serum-fed cells (Fig. $4 \mathrm{a}-\mathrm{c}$ ) without affecting the localization patterns of the TF proteins CEP164 and CEP83 (Fig. 4d). This TTBK2 accumulation triggered by INPP5E depletion was significantly suppressed by co-depleting PIPKI $\gamma$ (Fig. 4a,b). On the other hand, overexpression of the wild-type INPP5E almost completely inhibited TTBK2 recruitment in response to serum starvation, whereas the catalytic-defective INPP5E (D512/515W) related to Joubert syndrome ${ }^{33}$ had no such effect (Fig. $4 \mathrm{e}-\mathrm{g}$ ), indicating that the activity of INPP5E is required to inhibit TTBK2 recruitment. As a consequence of impaired TTBK2 recruitment to the M-centriole, serum-starvation-induced CP110 removal and ciliogenesis were both inhibited in INPP5E overexpressing RCTE cells (Fig. 4h-j) as well as IMCD3 cells (Supplementary Fig. 4a,b). In summary, the alterations of TTBK2 recruitment and CP110 removal caused by decreasing or increasing INPP5E activity (Fig. 4a,e) recapitulated those caused by increasing or decreasing PIPKI $\gamma$ activity (Fig. 3c,e), respectively. This leads to a conclusion that PIPKI $\gamma$ and INPP5E oppositely regulate the recruitment of TTBK2 to the M-centriole during the initiative step of ciliogenesis.

A centrosomal PtdIns(4)P pool inhibits TTBK2 recruitment. It was reported that binding to CEP164 is required for TTBK2 recruitment to the $\mathrm{M}$-centriole/basal body ${ }^{37}$. Because the protein level of either TTBK2 or CEP164 is not affected by manipulating the activity of PIPKI $\gamma$ (Fig. 3d,g) or INPP5E (Fig. 4c,g), we reason that the association between TTBK2 and CEP164 might be disturbed. As expected, both PIPKI $\gamma$ depletion (Fig. 5a) and INPP5E overexpression (Fig. 5b) led to substantially reduced association between TTBK2 and CEP164. These results, together with our data that the activities of PIPKI $\gamma$ (Fig. $3 \mathrm{e}-\mathrm{g}$ ) and INPP5E (Fig. 4e-g) are required for regulating TTBK2 recruitment, suggest that PtdIns(4)P (substrate of PIPKI $\gamma$ and product of INPP5E) and/or PtdIns $(4,5) \mathrm{P}_{2}$ (product of PIPKI $\gamma$ and substrate of INPP5E) may regulate the interaction between TTBK2 and CEP164. To test this, we first determined whether PtdIns(4)P or PtdIns $(4,5) \mathrm{P}_{2}$ presents at the centrosome. As shown in Fig. $5 \mathrm{c}$ validated PtdIns(4)P antibody ${ }^{30,31}$ (Supplementary Fig. 5a) nicely decorates the centrosome in serum-fed, non-ciliated cells; however, we did not observe specific centrosome accumulation of PtdIns(4,5) $\mathrm{P}_{2}$, PtdIns(3)P or PtdIns(5)P (Supplementary Fig. 5bd). The centrosomal PtdIns(4)P signal diminished when PIPKI $\gamma$ was overexpressed or serum starvation was applied (Fig. 5c,d), both conditions induce the TTBK2 recruitment to the $\mathrm{M}$-centriole. Furthermore, ectopic expression of the wild-type INPP5E restored PtdIns(4)P at the centrosome in serum-starved cells (Fig. $5 c, d$ ). These results, confirming the authenticity of this centrosomal PtdIns(4)P pool, indicate a negative correlation between the accumulation of PtdIns(4)P and the recruitment of TTBK2 at the centrosome. To further investigate whether this centrosomal PtdIns(4)P pool is physiologically relevant to the TTBK2 recruitment, we fused the pericentrin-AKAP450 centrosomal targeting (PACT) domain ${ }^{38}$ to the PtdIns(4)Pbinding P4M domain of $\operatorname{SidM}^{39}$ or PtdIns $(4,5) \mathrm{P}_{2}$-binding $\mathrm{PH}$ domain of PLC $\delta^{40}$. Then, we overexpressed these proteins in cells to achieve the spatial sequestration of $\operatorname{PtdIns}(4) \mathrm{P}$ or $\operatorname{PtdIns}(4,5) \mathrm{P}_{2}$ at the centrosome, respectively. Remarkably, only the PACT$\mathrm{P} 4 \mathrm{M}^{\text {SidM }}$, but not the PACT alone or PACT-PH ${ }^{\mathrm{PLC} \delta}$, resulted in an accumulation of TTBK2 at the centrosome in serum-fed cells (Fig. 5e,f), indicating that the depletion of centrosomal PtdIns(4)P favours TTBK2 recruitment in vivo. Considering the loss of PIPKI $\gamma$ and increase of INPP5E, both diminish the association between TTBK2 and CEP164 (Fig. 5a,b), we propose that PtdIns(4)P inhibits the TTBK2-CEP164 interaction.

PtdIns(4)P inhibits the CEP164-TTBK2 interaction. To fully understand the underlying mechanism, we examined whether TTBK2 or CEP164 can bind PtdIns(4)P and/or PtdIns(4,5) $\mathrm{P}_{2}$ in vitro by utilizing the PolyPIPosome liposomes ${ }^{41}$. Comparing with the control PolyPIPosomes that do not contain any PI species, PolyPIPosomes containing PtdIns(4)P, but not PtdIns $(4,5) \mathrm{P}_{2}$, interacts with both CEP164 and TTBK2 (Fig. 6a). Importantly, excessive PtdIns(4)P but not PtdIns $(4,5) \mathrm{P}_{2}$ significantly inhibited the interaction between TTBK2 and CEP164 in vitro (Fig. 6b), suggesting that PtdIns(4)P suppresses the TTBK2 recruitment mediated by CEP164. It has been reported that the $\mathrm{N}$ terminus of CEP164 binds to the $\mathrm{C}$ terminus of TTBK2 (ref. 15). When analysing the peptide sequences of TTBK2 and CEP164, we found consensus PI-binding motifs in CEP164 $\mathrm{N}$ terminus and TTBK2 
a
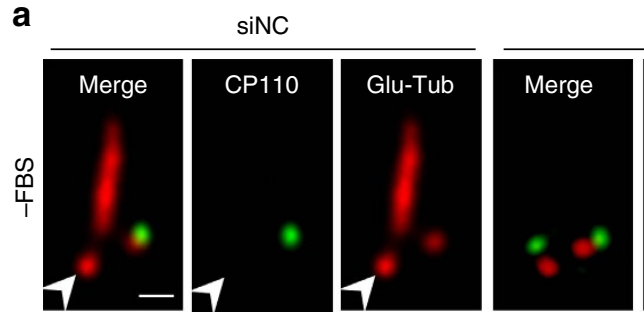

siPIPKI

b

siNC

siPIPKI $\gamma$
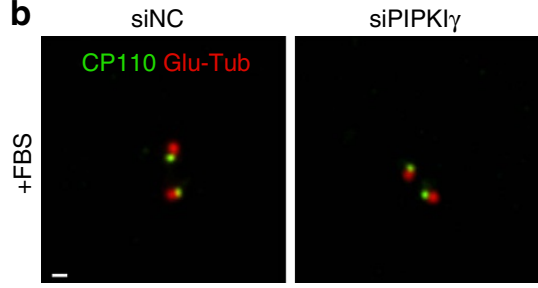

siCP110 + siPIPKl $\gamma$

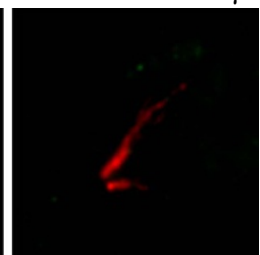

C

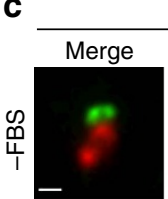

siNC
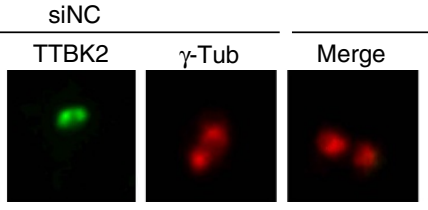

siPIPKl $\gamma$

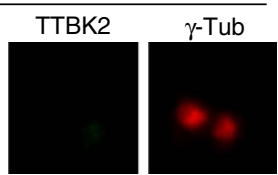

e
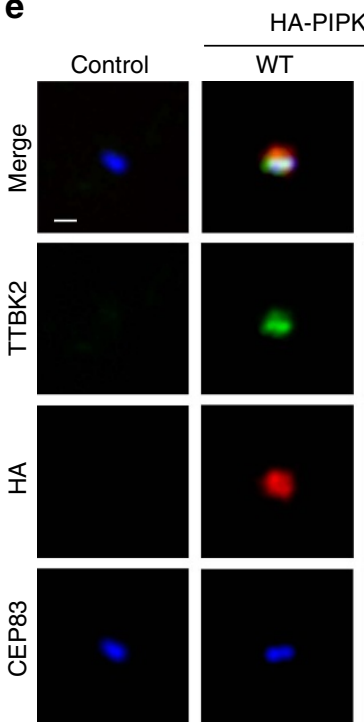

+FBS f

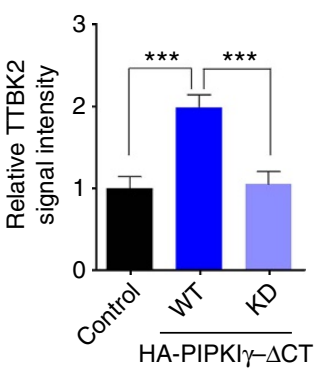

g
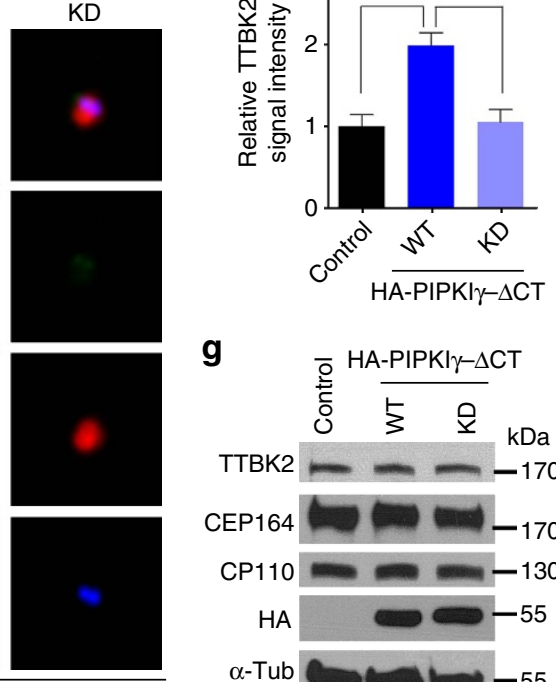

TTBK2 $--\frac{\mathrm{kDa}}{-170}$

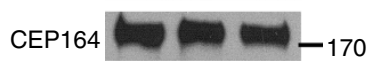

$\mathrm{CP} 110=-130$

$\mathrm{HA}$

$\alpha-$ Tub

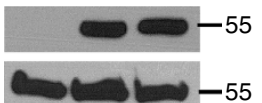

$\square \operatorname{siNC}$

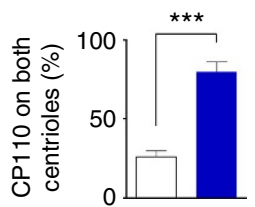

d
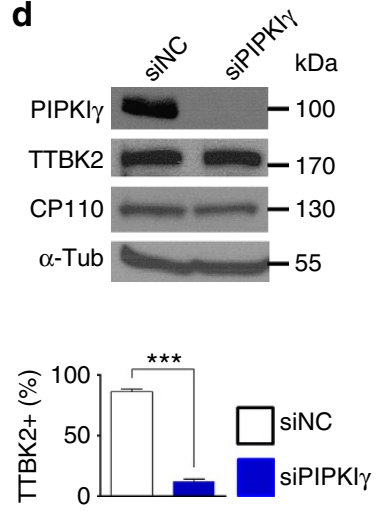

h Control HA-PIPKI $\gamma-\Delta \mathrm{CT}$
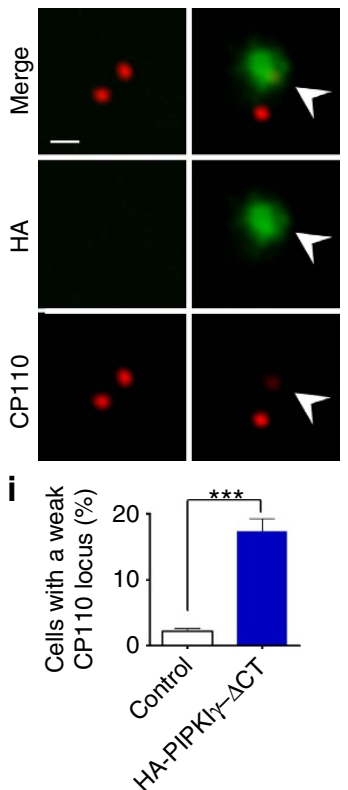

Figure 3 | PIPKI $\gamma$ is required for the removal of CP110 from and the recruitment of TTBK2 to the M-centriole/basal body. (a,c) RCTE cells were treated with control (siNC) or PIPKI $\gamma$-specific (siPIPKI $\gamma$ ) siRNA for $48 \mathrm{~h}$, serum starved ( - FBS) for additional $24 \mathrm{~h}$ and stained with indicated antibodies for IF microscopy. Percentage of cells $(n>200)$ with CP110 on both centrioles (a) or with TTBK2 at the M-centriole/basal body (c) was quantified from three independent experiments. Error bars represent s.d. (b) Serum-fed ( + FBS) RCTE cells were treated with siNC, siPIPKI $\gamma$ or siPIPKI $\gamma$ plus CP110-specific (siCP110) siRNA for $72 \mathrm{~h}$, and then subjected to IF microscopy to visualize polyglutamylated tubulin and CP110. (d) CP110 and TTBK2 protein levels are not changed in PIPKI $\gamma$-depleted cells. Cell lysates from control (siNC) or PIPKI $\gamma$-depleted (siPIPKI $\gamma$ ) cells were immunoblotted using indicated antibodies.

(e) Serum-fed RCTE cells stably expressing GFP-TTBK2 were transfected with empty vector (Control), wild-type (WT) or kinase-dead (KD) HA-PIPKI $\gamma \Delta$ CT for $8 \mathrm{~h}$. Then cells were subjected to IF microscopy to visualize TTBK2, HA tag and CEP83. (f) The fluorescence intensity of centrosomal TTBK2 in $>20$ control, HA-PIPKI $\gamma \Delta$ CT-WT or HA-PIPKI $\gamma \Delta$ CT-KD expressing cells was quantified from three independent experiments and plotted. Error bars represent s.e.m. ( $(\mathbf{g}$ ) Cell lysates from cells described in e were immunoblotted using indicated antibodies. $\alpha$-Tub, $\alpha$-tubulin. (h) CP110 was visualized by IF microscopy in serum-fed control or HA-PIPKI $\gamma \Delta$ CT-WT expressing cells. (i) Percentage of cells $(n>40)$ showing diminished CP110 signal at M-centrioles was quantified in each group and results from three independent experiments were plotted. Error bars represent s.d. Arrowheads indicate $M$-centrioles/basal bodies. ${ }^{\star \star \star} P<0.001$. Scale bars, $0.5 \mu \mathrm{m}$.

C terminus, respectively (Fig. 6c). These basic motifs are conserved throughout variant vertebrates (Supplementary Fig. 6a,b), suggesting that these motifs may exercise important function. We mutated the 9 lysine in the PI-binding motif of CEP164 to alanine (CEP164-9A) and deleted the basic motif from TTBK2 (TTBK2- $\triangle \mathrm{PI}$ ) (Fig. 6c). Both mutations abolished 
a

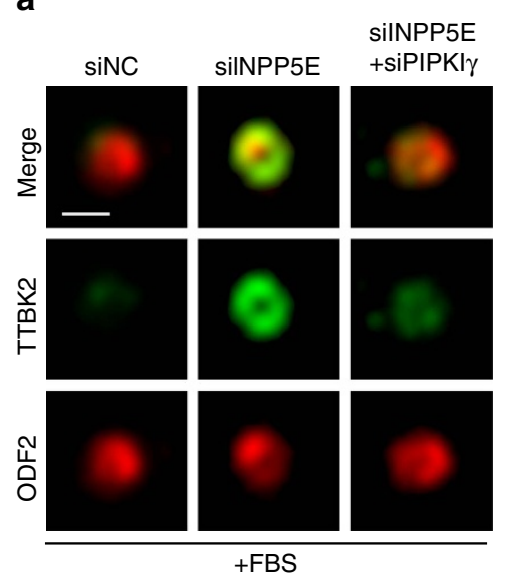

e
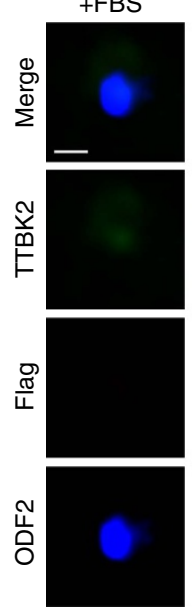

b

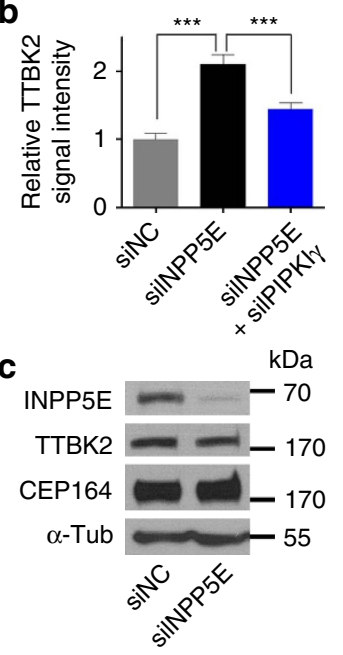

d
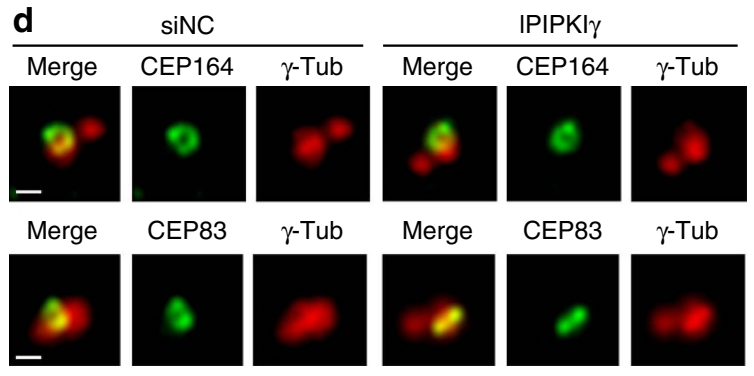

h
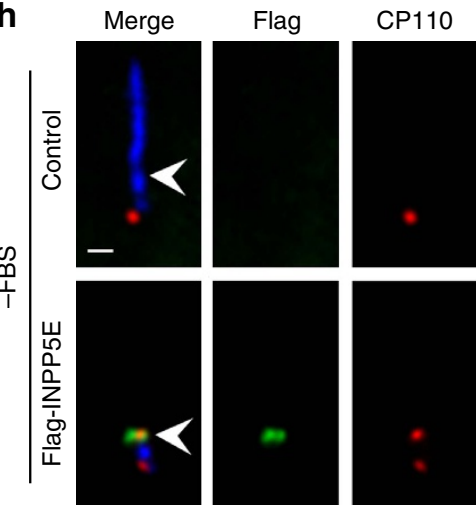

Ac-Tub

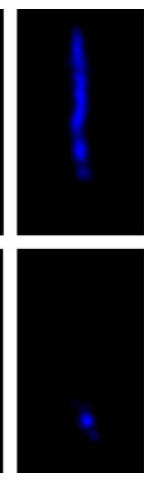

g

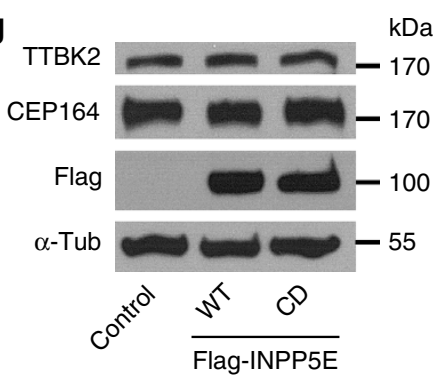

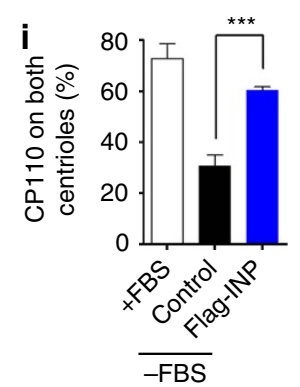

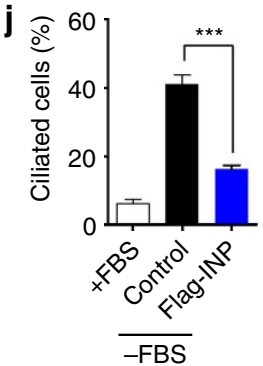

Figure 4 | INPP5E inhibits the association of TTBK2 with the M-centriole/basal body. (a,b) TTBK2 was visualized by IF microscopy in serum-fed RCTE cells $72 \mathrm{~h}$ post transfection of the negative control (siNC), INPP5E-specific (silNPP5E) and/or PIPKI $\gamma$-specific (siPIPKI $\gamma$ ) siRNAs. ODF2 was used as a centrosome marker. (b) The centrosomal TTBK2 intensity in cells $(n>30)$ of each group was quantified and plotted from three independent experiments. $(\mathbf{c}, \mathbf{d})$ RCTE cells treated with siNC or silNPP5E were collected for immunoblotting (c) or IF microscopy (d) with indicated antibodies. $\alpha$-Tub, $\alpha$-tubulin. $(\mathbf{e}, \mathbf{f})$ RCTE cells were transfected with empty vector (Control), wild type (WT) or catalytic defective (CD) Flag-INPP5E for $8 \mathrm{~h}$, and serum starved for additional $8 \mathrm{~h}$ before stained with indicated antibodies. In each group, the intensity of centrosomal TTBK2 signal in $>30$ cells was quantified from at least three independent experiments and plotted. Error bars represent s.e.m. (g) Cells transfected with empty vector (Control), WT or CD Flag-INPP5E were collected for immunoblotting with indicated antibodies. (h-j) RCTE cells transfected with empty vector (Control) or wild-type Flag-INPP5E for $24 \mathrm{~h}$ were serum-starved for additional $36 \mathrm{~h}$, and then stained with appropriate antibodies. Percentage of cells with CP110 on both centrioles (i; $n>100)$ or with primary cilium ( $\mathbf{j} ; n>200$ ) was quantified in each group. Results from at least three independent experiments were plotted. Error bars represent s.d. ${ }^{\star \star \star} P<0.001$. Scale bars, $0.5 \mu \mathrm{m}$.

the interaction with PtdIns(4)P-containing PolyPIPosomes (Supplementary Fig. 6c,d), which confirms that these motifs indeed mediate the binding of PtdIns(4)P to CEP164 and TTBK2, respectively. More importantly, the interactions between the wild-type CEP164 and TTBK2- $\triangle$ PI, or between CEP164-9A and the wild-type TTBK2 are both stronger than the interaction between the wild-type proteins; and as expected, CEP164-9A and TTBK2- $\Delta$ PI exhibited the strongest interaction (Fig. 6d). These results indicate that both CEP164 and TTBK2 can bind PtdIns(4)P and this binding inhibits the interaction between CEP164 and TTBK2.

Consistent with our results from in vitro protein-lipid and protein-protein interaction assays, the PtdIns(4)P-bindingdeficient CEP164-9A, but not the wild-type CEP164, could recruit the endogenous TTBK2 to the centrosome independent of serum starvation (Fig. 7a,b). In addition, overexpression of
CEP164-9A restored the serum-starvation-induced TTBK2 recruitment to the $\mathrm{M}$-centriole/basal body in PIPKI $\gamma$-depleted cells (Fig. 7c,d). These results reconcile with our previous observations resulted from manipulating the activity of PIPKI $\gamma$ or INPP5E, and indicate an interesting possibility that PIPKI $\gamma$ and INPP5E coordinate PtdIns(4)P homoeostasis at the distal end of the M-centriole/basal body. Insomuch as TTBK2 was established as a critical regulator for the removal of CP110 and axoneme elongation ${ }^{14}$, it is vital to understand how TTBK2 accumulation at the $\mathrm{M}$-centriole/basal body is regulated during the initiating stage of ciliogenesis. Our current results suggest a working model as summarized in Fig. 7e. In non-ciliated cells, INPP5E resides at the distal end of the M-centriole to maintain high level of regional PtdIns(4)P through hydrolyzing $\operatorname{PtdIns}(4,5) \mathrm{P}_{2}$ presumably generated by PIPKI $\gamma$, and these local PtdIns(4)P molecules bind CEP164 and inhibit its interaction with 
a

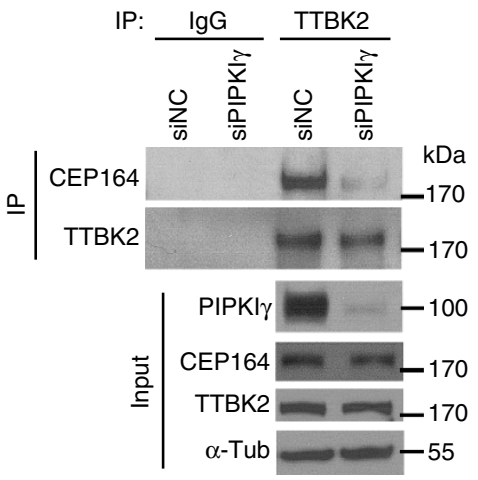

b

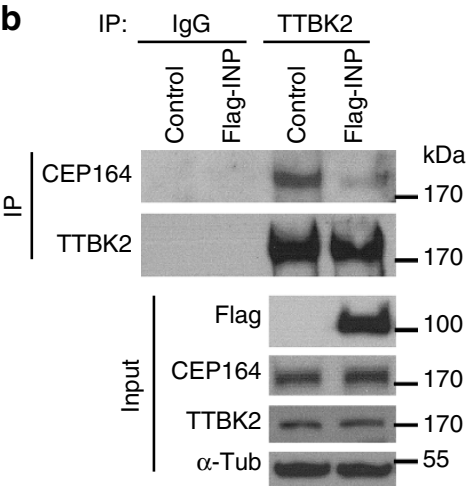

C

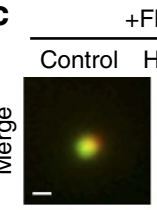

+FBS
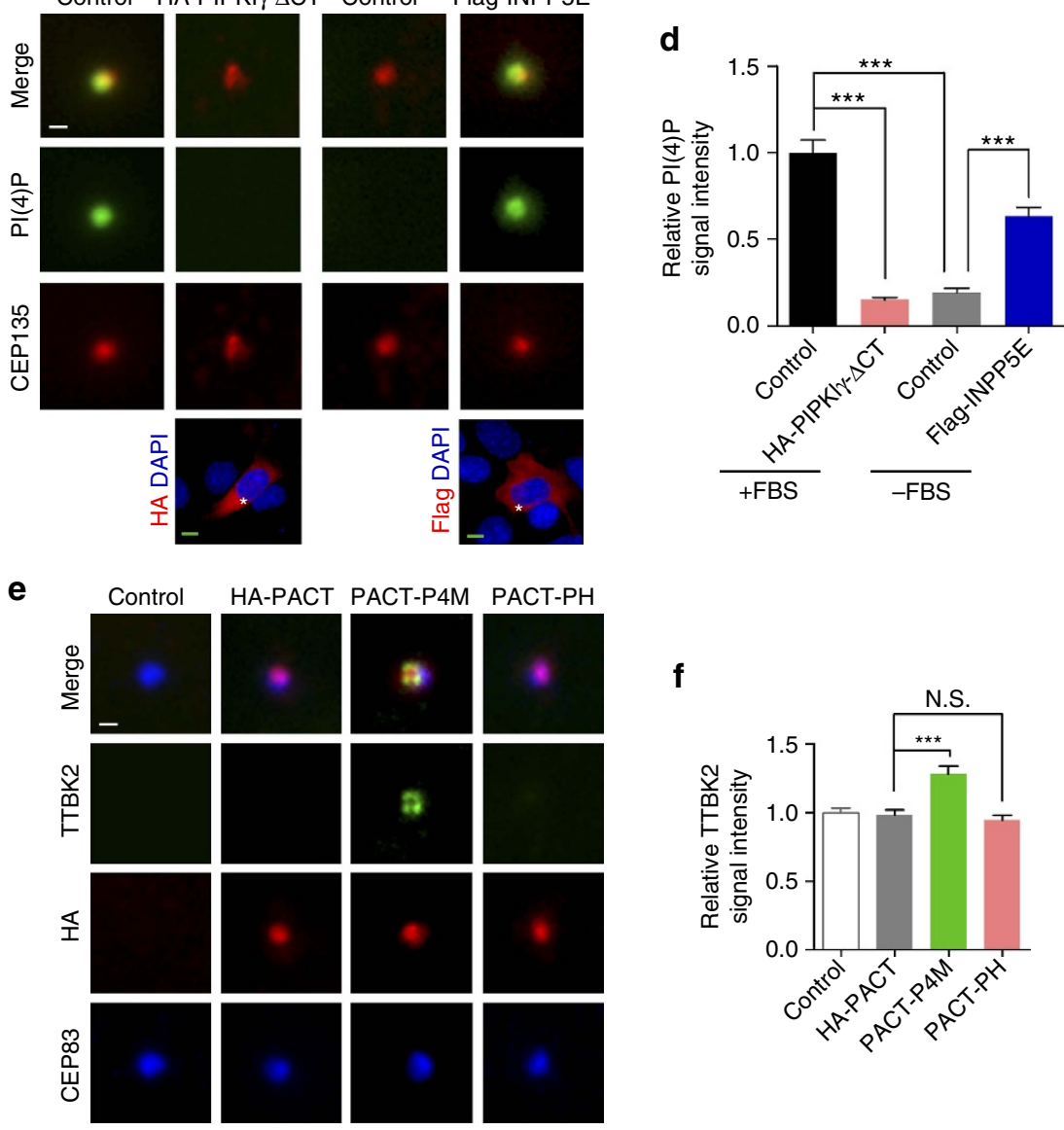

$\mathbf{f}$

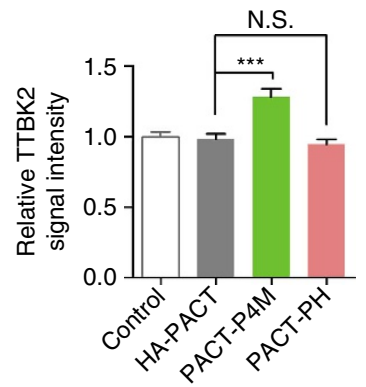

+ FBS

Figure 5 | PIPKI $\gamma$ and INPP5E coordinate the association between TTBK2 and CEP164 by regulating a centrosomal Ptdlns(4)P pool. (a,b) HEK293T cells transfected with indicated siRNAs (a) or plasmids (b) were subjected to immunoprecipitation (IP) with anti-TTBK2 antibody or normal rabbit lgG (IgG). Flag-INP, Flag-INPP5E. The resulting precipitates and cell lysates (Input) were immunoblotted with indicated antibodies. $\alpha$-Tub, $\alpha$-tubulin. (c) IMCD3 cells were transfected with empty vector (Control), HA-PIPKI $\gamma \Delta C$ T or Flag-INPP5E. Without ( + FBS) or with 6- h serum starvation ( - FBS, most cells were not yet ciliated under this condition), each group of cells was subjected to IF microscopy to visualize Ptdlns(4)P (PI(4)P, green), CEP135 (red), HA-tag (far-red) and nuclei (blue, DAPI staining). Stars indicate transfected cells. (d) In each group in c, the intensity of centrosomal Ptdlns(4)P signal in $>20$ cells was quantified and plotted from at least three independent experiments. (e,f) RCTE cells stably expressing GFP-TTBK2 were transfected with HA-tagged PACT, PACT-P4M SidM (PACT-P4M) or PACT-PHPLC (PACT-PH) for $24 \mathrm{~h}$. Cells were then processed for IF microscopy with antibodies against TTBK2 (green), HA-tag (red) and CEP83 (far-red, pseudo-colored blue). (f) The fluorescence intensity of centrosomal TTBK2 was quantified in $>20$ cells of each group. Results from three independent experiments were plotted. Error bars represent s.e.m. ${ }^{\star \star \star} P<0.001$. Scale bars, $5 \mu \mathrm{m}$ (green); Scale bars, $0.5 \mu \mathrm{m}$ (white).

TTBK2. After serum starvation, INPP5E is removed from the basal body through unknown mechanism, which leads to a decrease of local PtdIns(4)P level at the distal end of the basal body. This facilitates the association of TTBK2 with CEP164, and in turn triggers CP110 removal and downstream axoneme elongation.

\section{Discussion}

In spite of only being a small fraction of cellular phospholipids, PIs regulate almost many aspects of cell physiology by spatiotemporally regulating variants cellular processes 19,42 Recently, the role of PI signalling in the context of primary 
a
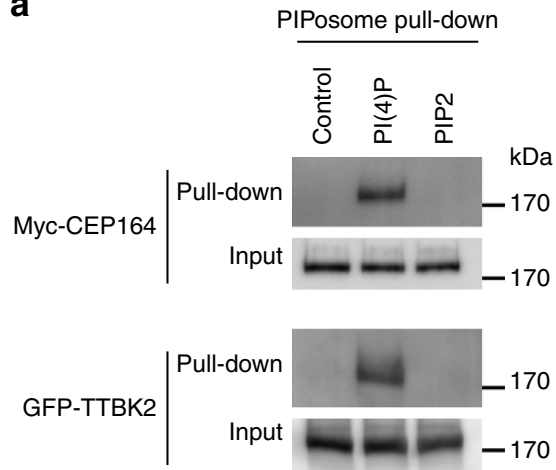

C

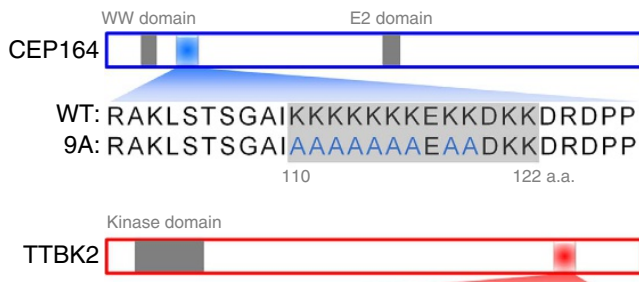

WT: PRPPPGKPPVRPGVEARLRRYKVLGSSN $\triangle \mathrm{PI}$ PRPPPGKPPV YKVLGSSN

b

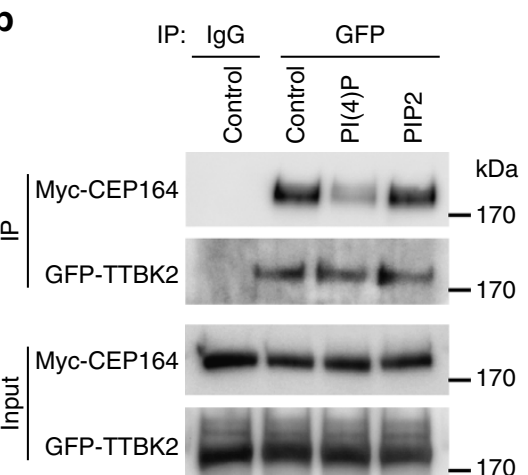

d

Myc-CEP164: WT 9A EV WT

GFP-TTBK2: WT $\triangle \mathrm{PI}$ WT $\triangle \mathrm{PI}$ WT EV

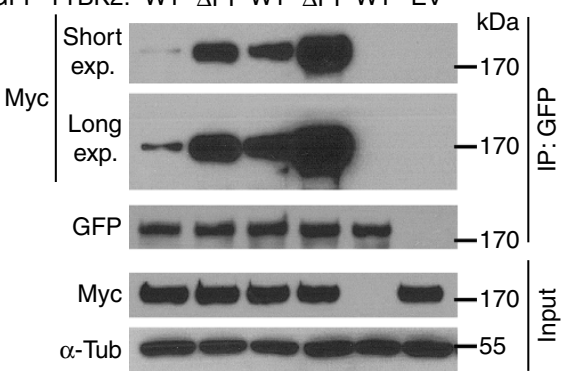

Figure 6 | Ptdlns(4)P binds to CEP164 and TTBK2 and inhibits their interaction in vitro. (a) Cell lysates prepared from HEK293T cells overexpressing Myc-CEP164 or GFP-TTBK2 were subjected to PolyPIPosome (PIPosome) pull-down assay with control PolyPIPosomes or PolyPIPosomes containing Ptdlns(4)P (PI(4)P) or Ptdlns(4,5) $\mathrm{P}_{2}$ (PIP2). The resulting precipitates and cell lysates (Input) were immunoblotted with indicated antibodies.

(b) HEK293T cells co-transfected with Myc-CEP164 and GFP-TTBK2 were subjected to immunoprecipitation (IP) with GFP antibody or normal mouse $\lg (\operatorname{lgG})$, in the absence or presence of PI(4)P or PIP2. The resulting precipitates were examined by immunoblotting with indicated antibodies.

(c) Schematic illustration of the consensus PI-binding motifs in CEP164 and TTBK2. The PI-binding-deficient CEP164 or TTBK2 were generated by mutating or deleting the basic amino acids in these motifs, respectively. (d) HEK293T cells co-transfected with indicated combination of wild type (WT) or mutated (9A) Myc-CEP164 and wild type or mutated ( $\triangle$ PI) GFP-TTBK2 were subjected to IP with anti-GFP antibody. The resulting precipitates and cell lysates (Input) were immunoblotted with indicated antibodies. EV, empty vector; long exp., long exposure; ; short exp., short exposure; $\alpha$-Tub, $\alpha$-tubulin.

cilia emerges and starts to be appreciated. INPP5E, previously shown to support the stability of pre-existing primary cilia $^{23,33}$, is also critical to maintain a $\operatorname{PtdIns}(4,5) \mathrm{P}_{2}$-low environment in ciliary membrane for the activation of Hedgehog signalling ${ }^{30,31}$. Here our results demonstrate a novel role for INPP5E in regulating ciliogenesis. We find that INPP5E resides at the distal end of the M-centriole in non-ciliated cells, in addition to its reported localization in the ciliary lumen in ciliated cells. By keeping a PtdIns(4)P-high microenvironment, active INPP5E at the M-centriole inhibits TTBK2 recruitment and the subsequent CP110 removal and ciliogenesis. These results indicate that the exclusion of INPP5E from the M-centriole is essential for ciliogenesis. Signals initiating ciliogenesis, such as serum starvation, very likely triggers the translocation of INPP5E from the M-centriole to the ciliary lumen. It has been shown that ARL13B, PDE6D and CEP164 are required for the ciliary targeting of INPP5E (ref. 43). Garcia-Gonzalo et al. ${ }^{31}$ also showed that TZ components TCTN1 and MKS complex confine INPP5E within the cilium. It would be interesting to investigate whether any of these proteins regulate the dissociation of INPP5E from the M-centriole at the initiating stage of ciliogenesis, and if so, what the underlying mechanism is.

In addition to INPP5E, our results also reveal an innovative role for PIPKI $\gamma$ in promoting ciliogenesis by depleting centrosomal PtdIns(4)P. This is achieved via a novel mechanism that PtdIns(4)P binds to CEP164 and TTBK2 and inhibits the CEP164-TTBK2 interaction, therefore prevents TTBK2 recruitment. It is worth noting that although artificially decreasing centrosomal PtdIns(4)P by ectopically expressed PIPKI $\gamma$ is sufficient to promote TTBK2 association with the M-centriole, it fails to completely remove $\mathrm{CP} 110$ and initiates axoneme assembly. This suggests that TTBK2, after being recruited to the M-centriole, might need to be further activated by certain PIPKI $\gamma$-independent mechanism to achieve the complete removal of CP110 that is required for axoneme elongation. Nevertheless, our results indicate that PIPKI $\gamma$ and INPP5E coordinates the centrosomal homoeostasis of PtdIns(4)P during ciliogenesis. Given the importance of PI signalling in ciliogenesis initiation identified here and the identification of various PI 5-phosphatases (such as INPP5E, INPP5B and OCRL) as causal loci for ciliopathies, we reason that PIPKI $\gamma$ malfunction may also be correlated with ciliopathies. A kinase-dead mutation in PIPKI $\gamma$ leads to neurodegeneration in human ${ }^{44}$ and PIPKI $\gamma$-null mice that exhibit embryonic lethality at E11.5 with defects in neuronal tube closure ${ }^{45}$, both of which are likely ciliopathy phenotypes. Due to the existence of multiple PIPKI $\gamma$ splicing isoforms, further in vivo investigations are needed to dissect the exact correlation between each PIPKI $\gamma$ isoform with primary cilia and ciliopathies.

Here we report a functional centrosomal PtdIns(4)P pool that is coordinated by INPP5E and PIPKI $\gamma$. All of the PtdIns(4) P-enriched endomembranes, such as the Golgi complex, Golgiderived carriers, as well as the recycling endosome ${ }^{46-48}$, likely contribute to ciliogenesis by supporting IFT20-dependent vesicle transport ${ }^{49}$ or composing the ciliary vesicles that attach to the 
a

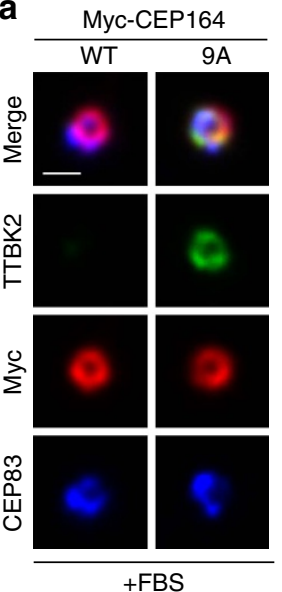

e

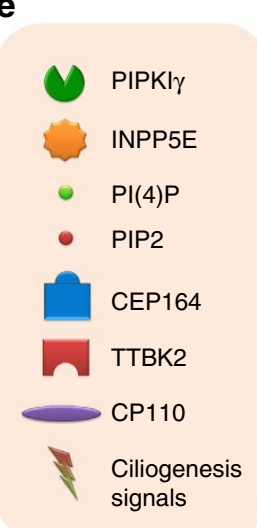

b

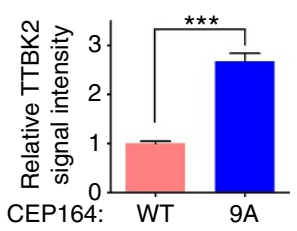

d

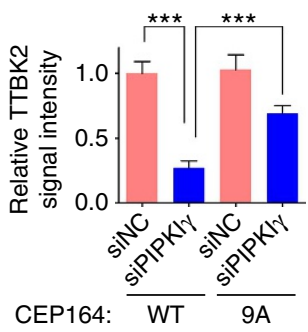

C

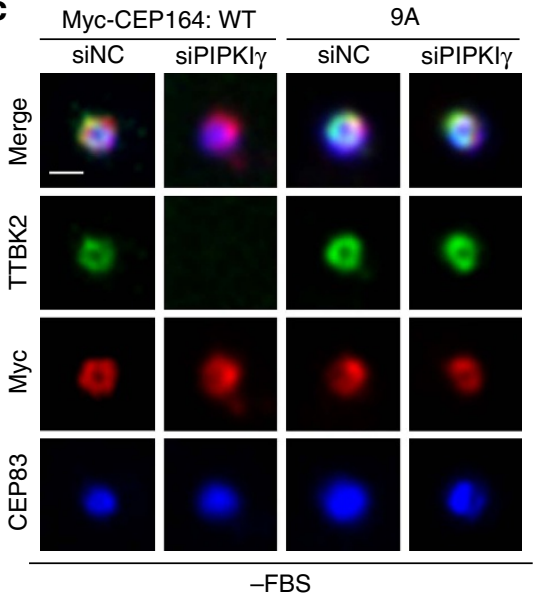

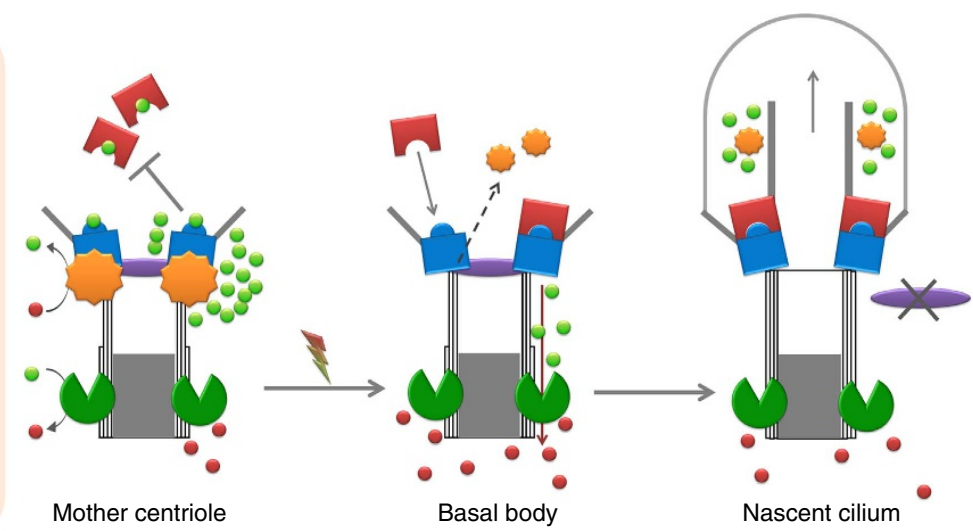

Figure 7 | Centrosomal PtdIns(4)P homoeostasis at the $\mathbf{M}$-centriole/basal body. (a,b) RCTE cells stably expressing GFP-TTBK2 were transfected with wild type (WT) or mutated Myc-tagged CEP164 (9A) for $24 \mathrm{~h}$ and subjected to IF microscopy after stained with indicated antibodies. (b) The fluorescence intensity of centrosomal TTBK2 in $>20$ cells of each group quantified from three independent experiments and plotted. (c,d) RCTE cells stably expressing GFP-TTBK2 were transfected with negative control (siNC) or PIPKl $\gamma$ siRNA (siPIPKl $\gamma$ ) for $24 \mathrm{~h}$ followed by transfection with wild type (WT) or mutant Myc-CEP164 (9A) for another $24 \mathrm{~h}$. After being serum starved for additional $24 \mathrm{~h}$ to induce the translocation of TTBK2 to the M-centriole/basal body, these cells were stained with indicated antibodies for IF microscopy. (d) The fluorescence intensity of centrosomal TTBK2 was quantified in each group $\left(n>20\right.$ cells). Results from three independent experiments were analysed and plotted using Excel. Error bars represent s.e.m. ${ }^{\star \star \star} P<0.001$. Scale bars, $0.5 \mu \mathrm{m}$. (e) Schematic model summarizing the results of current study. In proliferating cells, INPP5E localizes at the distal end of the M-centriole and plays a key role in repelling TTBK2 by maintaining a local Ptdln(4)P (PI(4)P) pool that inhibits the interaction of TTBK2 with CEP164. In quiescent cells when INPP5E leaves the centrosome, the centrosomal PIPKI $\gamma$ promotes the TTBK2 recruitment and the initiation of ciliary axoneme elongation by consuming the $\mathrm{PI}(4) \mathrm{P}$ at the centrosome. PIP2, Ptdlns $(4,5) \mathrm{P}_{2}$.

distal end of the M-centriole/basal body to form the ciliary membrane $\mathrm{s}^{50}$. Since we were not able to co-stain any membrane marker with the PtdIns(4)P antibody using immunofluorescence microscopy, whether the centrosomal PtdIns(4)P we observed is PtdIns(4)P-containing vesicles should be confirmed by using immunoelectron microscopy. It is also possible that unknown ciliary PI-transfer protein(s) $)^{51}$ shuttles PtdIns(4)P and/or PtdIns $(4,5) \mathrm{P}_{2}$ between the distal and proximal ends of the $\mathrm{M}$-centriole where INPP5E and PIPKI $\gamma$ reside, respectively.

Similar as we reported here and previously ${ }^{32}$, Chavez et al. ${ }^{30}$ also observed PIPKI $\gamma$ at the ciliary base. However, we failed to detect outstanding PtdIns $(4,5) \mathrm{P}_{2}$ accumulation at the centrosome or basal body using both specific antibody and biosensor, although our results indicate that PIPKI $\gamma$ should generate PtdIns $(4,5) \mathrm{P}_{2}$ by phosphorylating the centrosomal PtdIns(4)P during ciliogenesis initiation. This could be due to inappropriate technique, or the PtdIns $(4,5) \mathrm{P}_{2}$ produced by PIPKI $\gamma$ is quickly consumed or masked by centrosomal/ciliary base $\operatorname{PtdIns}(4,5) \mathrm{P}_{2}$ effector or binding proteins like MARCKS ${ }^{52}$. In addition to supporting ciliogenesis, PIPKI $\gamma$ in ciliated cells may help maintain the proximal PtdIns $(4,5) \mathrm{P}_{2}$-containing domain of the ciliary membrane, which is indispensible for ciliary signalling by regulating the transport of ciliary cargos, such as in the case of TULP3-mediated transport of Gpr161 and hedgehog signalling inhibition $^{31}$. Moreover, multiple TZ proteins contain C2 domain $^{13}$ that could bind PIs ${ }^{53}$. Whether these proteins are regulated by PI signalling should be investigated. Interestingly, a recent work indicates that $\mathrm{C} 2$ domain-containing $\mathrm{TZ}$ protein MKS5 establishes a TZ zone that limits the abundance of PtdIns $(4,5) \mathrm{P}_{2}$ within cilia and is required for ciliary signalling ${ }^{54}$, indicating complex interactions between PIs, PI enzymes and PI effectors in the context of primary cilium. After all, how ciliumassociated PI metabolizing enzymes orchestrate the dynamic, spatiotemporally regulated homoeostasis of variant PIs to support ciliogenesis and cilium function is a fascinating question to explore in the future. Our current work demonstrates the PIPKI $\gamma$-INPP5E signalling nexus and the novel role of PtdIns(4)P homoeostasis in the initiation of ciliogenesis, which provides attractive targets for future small-molecule intervention of human ciliopathies related to PIPKI $\gamma$ and/or INPP5E. 


\section{Methods}

Antibodies. Rabbit polyclonal antibodies against the following proteins were used: CEP83 (HPA038161), CEP164 (SAB3500022) and TTBK2 (HPA018113) (Sigma); CP110 (A301-343A, Bethyl Laboratories); CEP135 (ab75005), Actin (ab8227) (Abcam); FBF1 (11531-1-AP), TCTN1 (15004-1-AP), SCLT1 (14875-1-AP), MKS1 (16206-1-AP), INPP5E (17797-1-AP) and IFT88 (13967-1-AP) (Proteintech). Rabbit antibodies against PIPKI $\gamma$, PIPKI $\alpha$ and PIPKI $\beta$ were described previously ${ }^{32}$. Mouse antibodies against the following proteins were used: Centrin (20H5) (kindly provided by Dr Jeffrey Salisbury, Mayo Clinic); polyglutamylated tubulin (ALX-804-885-C100, Enzo Life Sciences); ODF2 (H00004957-M01, Abnova); acetylated $\alpha$-tubulin (T7451), $\alpha$-tubulin (T9026), $\gamma$-tubulin (T6557), FLAG (F1804) and HA (H3663) (Sigma); GFP (A-11120, Invitrogen); PI(4)P (Z-P004) and PI(4,5)P2 (Z-P045) (Echelon). Mouse monoclonal NPHP1 antibody (IgG 1) was generated at Abmart. For western blots, we used a 1:5,000 dilution for antibodies except for those against $\alpha$-tubulin and actin $(1: 20,000)$. For immunofluorescence microscopy, a 1:500 dilution for antibodies was used except for those against NPHP1 (1:50), acetylated $\alpha$-tubulin, polyglutamylated tubulin, centrin $(1: 2,000)$, PtdIns(4)P and PtdIns(4,5) $\mathrm{P}_{2}$ (1:100). Uncropped western blots are shown in Supplementary Fig. 7.

DNA constructs and siRNAs. The GFP-TTBK2 construct was kindly provided by Dr Kathryn Anderson (Sloan-Kettering Institute, New York, NY). The Flag-INPP5E construct was a kind gift from Dr Seongjin Seo (University of Iowa, Iowa City, IA). We obtained the following constructs from Addgene: Myc-CEP164 (Addgene plasmid \#41148, from Dr Erich Nigg), GFP-ING2 (pHD) (Addgene plasmid \#21589, from Dr Junying Yuan), GFP-FYVE (EEA1) (Addgene plasmid \#36096, from Dr Scott Emr). We sub-cloned the FYVE(EEA1) into pEGFP-C1 vector. PIPKI $\gamma$ and PIPKI $\gamma \Delta \mathrm{CT}$ were amplified from $\mathrm{CDNAs}$ and sub-cloned into pCMV-HA vector ${ }^{32}$. Inactive PIPKI $\gamma(\mathrm{D} 253 / 316 \mathrm{~A})^{55,56}$, INPP5E (D512/515W) ${ }^{33}$ and PI-binding-deficient CEP164 and TTBK2 mutants (Myc-CEP164-9A and GFP-TTBK2- $\Delta$ PI) were generated by using a QuikChange Site-Directed Mutagenesis Kit (Agilent Technologies). All constructs were verified by DNA sequencing.

siRNA duplexes were introduced into cells with Lipofectamine RNAiMAX (Invitrogen), following the manufacturer's manual. Typically after $72 \mathrm{~h}$ of siRNA transfection, cells were collected for further analysis by western blot or immunofluorescence microscopy. siRNA targeting INPP5E (ON-TARGETplus SMARTpool, \#L-020852-00-0005) and RNAi Negative Control were purchased from GE Healthcare Dharmacon. PIPKI $\gamma$, PIPKI $\alpha$ and PIPKI $\beta$ siRNA oligonucleotides were obtained from Invitrogen. PIPKI $\gamma$-O1 and PIPKI $\gamma$-O2 are siRNAs targeting human PIPKI $\gamma$.

PIPKI $\gamma$-O1, 5'-GCGTGGTCAAGATGCACCTCAAGTT-3';

PIPKI $\gamma$-O2: $5^{\prime}$-GCTACTACATGAACCTCAACCAGAA- $3^{\prime}$

mouse PIPKI $\gamma$ : PIPKI $\gamma$-O3, 5'-GCGAGAGAGAGGATGTGCAGTATGA-3'.

Human PIPKI $\alpha$ : 5'-TTGAAAGGTGCCATCCAGTTAGGCA-3';

Human PIPKI $\beta$ : 5'-CAGCAAAGGGTTACCTTCCAGTTCA-3';

Cell culture and transfection. NIH3T3 and HEK293T cells were cultured in DMEM containing 10\% foetal bovine serum (FBS). hTERT-RPE-1 (RPE-1), inner medullary collecting duct (IMCD3) and RCTE cells were cultured in DMEM/F-12 containing $10 \%$ FBS.

For plasmid transfection, X-tremeGENE 9 (Roche) were used following the manufacturer's manual. To generate RCTE cell line stably expressing GFP-TTBK2, RCTE cells were passed into complete growth medium containing $800 \mu \mathrm{g} \mathrm{ml}^{-1}$ G418 (MediaTech) for selection, and maintained in medium containing $400 \mu \mathrm{g} \mathrm{ml}^{-1} \mathrm{G} 418$.

Immunofluorescence microscopy. For indirect immunofluorescence, cells were grown on glass coverslips and fixed with ice-cold methanol or paraformaldehyde for $10 \mathrm{~min}$, then permeabilized and immune-stained with appropriate antibodies. Fluorescence images were acquired using Nikon TE2000-U with Metamorph software (Molecular Devices). Immunofluorescence staining of PtdIns(4)P and $\operatorname{PtdIns}(4,5) \mathrm{P}_{2}$ was performed following published methods $\mathrm{s}^{30,31,57}$. The specificity of the PtdIns(4)P and PtdIns $(4,5) \mathrm{P}_{2}$ antibodies was verified by pre-absorbing the antibody with PolyPIPosomes containing 5\% of PtdIns(4)P or PtdIns $(4,5) \mathrm{P}_{2}$ (Echelon) for $1 \mathrm{~h}$ at room temperature before incubation with samples.

Three dimensional structured illumination microscopy was performed following standard protocol. Briefly, an ELYRA Super-resolution Microscopy system (Zeiss) equipped with an alpha 'Plan-Apochromat' $100 \times / 1.46$ Oil DIC oil immersion objective and an Andor iXon 885 EMCCD camera was used to acquire raw images following standard protocols. Sections were acquired at $0.125-\mathrm{mm}$ $\mathrm{z}$-steps. Colour channels were aligned using alignment parameter from control measurements with $0.5-\mu \mathrm{m}$ diameter multispectral fluorescent beads (Zeiss). Structured illumination reconstruction and image processing were performed with the ZEN software package (Zeiss). Final image processing was done using Adobe Photoshop (Adobe).

Immunoprecipitation and PolyPIPosome pull-down assay. Immunoprecipitation was performed using HEK293T cell lysate $48 \mathrm{~h}$ post transfection, in IP buffer
(20 mM Hepes-KOH, pH 7.2, $10 \mathrm{mM} \mathrm{KCl,} 1.5 \mathrm{mM} \mathrm{MgCl}$, 1 mM EDTA, $1 \mathrm{mM}$ EGTA, $150 \mathrm{mM} \mathrm{NaCl}, 0.5 \% \mathrm{NP}-40$ ), with Complete Protease Inhibitor Cocktail (Roche) and PhosSTOP Phosphatase Inhibitor Cocktail (Roche) added following the manufacturer's manuals.

For immunoprecipitation assay in the presence of PIs, HEK293T cells co-transfected with Myc-CEP164 and GFP-TTBK2 were subjected to immunoprecipitation with GFP antibody and Protein G Sepharose 4 Fast Flow beads (GE Healthcare) overnight at $4^{\circ} \mathrm{C}$. After being washed, the protein-bound beads were incubated with $25 \mu \mathrm{M}$ diC8-PtdIns(4)P or diC8-PtdIns(4,5) $\mathrm{P}_{2}$ (Echelon) for additional $3 \mathrm{~h}$ at $4{ }^{\circ} \mathrm{C}$. The resulting precipitates were examined by immunoblotting with appropriate antibodies.

For PolyPIPosome pull-down assay, HEK293T cells were transfected with Myc-CEP164 or GFP-TTBK2 for $48 \mathrm{~h}$, and lysed in PolyPIPosome-binding buffer (50 mM Tris, pH 7.4, $150 \mathrm{mM} \mathrm{NaCl}, 1 \mathrm{mM}$ EGTA, $1 \mathrm{mM} \mathrm{MgCl}, 1 \mathrm{mg} \mathrm{ml}^{-1}$ bovine serum albumin, $0.2 \mathrm{mM} \mathrm{CaCl}_{2}, 5 \mathrm{mM} \mathrm{KCl}, 0.05 \% \mathrm{NP}-40$ ) on ice. The cell lysate was incubated with $50 \mu \mathrm{M}$ biotin-tagged control or PI-containing PolyPIPosomes (containing 5\% variant PI, Echelon Biosciences) overnight at $4{ }^{\circ} \mathrm{C}$, then incubated with Streptavidin Agarose beads (Thermo Fisher Scientific) for additional $1 \mathrm{~h}$. After being washed for three times with PolyPIPosome-binding buffer, the samples were analysed by immunoblotting with appropriate antibodies.

Electron microscopy. For transmission electron microscopy, IMCD3 cells treated with PIPKI $\gamma$ siRNA, or negative control siRNA were grown on coverslips. After $72 \mathrm{~h}$, the cells were serum starved to induce primary ciliogenesis for additional $24 \mathrm{~h}$. The cells were then fixed overnight using Trump's fixative (Electron Microscopy Sciences), scraped off the coverslips and spun into pellets. Embedding and thin sectioning of cell samples were performed according to standard procedures at the Electron Microscopy Core Facility, Mayo Clinic. Specimens were observed under a JEOL 1400 transmission electron microscope (JEOL) operating at $80 \mathrm{kV}$.

For scanning electron microscopy, IMCD3 cells treated with appropriate siRNAs were grown on coverslips. After $72 \mathrm{~h}$, cells were serum starved for additional $24 \mathrm{~h}$ to induce primary ciliogenesis. These cells were then fixed overnight in Trump's fixative and processed at the Mayo Clinic Electron Microscopy Core Facility according to standard procedures. Specimens were observed under a scanning electron microscope (Hitachi, S-4700).

Dye-filling assay. A dye-filling assay was performed using an established protocol ${ }^{58}$. Briefly, worms were washed off the culturing plate with $\mathrm{M} 9$ buffer ( $3 \mathrm{gl}^{-1} \mathrm{KH}_{2} \mathrm{PO}_{4}, 6 \mathrm{gl}^{-1}, \mathrm{Na}_{2} \mathrm{HPO}_{4}, 5 \mathrm{gl}^{-1} \mathrm{NaCl}$ and $1 \mathrm{mM} \mathrm{MgSO}$ ), collected by centrifugation at $500 \mathrm{~g}$ for $1 \mathrm{~min}$, washed once with $\mathrm{M} 9$ buffer, and then incubated in diluted DiI dye (Molecular Probes, D-282; 1:200 dilution in M9 buffer of the $2 \mathrm{mg} \mathrm{ml}^{-1}$ stock solution in dimethyl formamide) for $1 \mathrm{~h}$ at room temperature. After incubation, worms were washed at least three times with M9, transferred to an NGM plate without a bacterial lawn, and observed under a fluorescence microscope (Zeiss, M2Bio).

Statistical analyses. Results are presented as mean plus s.d. or s.e.m., as specified in each figure legend. Significance was calculated by double-tailed $t$-test using Excel (Microsoft). ${ }^{\star} P<0.05,{ }^{* *} P<0.01$ and ${ }^{* * *} P<0.001$ were considered as statistically significant differences.

\section{References}

1. Gerdes, J. M., Davis, E. E. \& Katsanis, N. The vertebrate primary cilium in development, homeostasis, and disease. Cell 137, 32-45 (2009).

2. Goetz, S. C. \& Anderson, K. V. The primary cilium: a signalling centre during vertebrate development. Nat. Rev. Genet. 11, 331-344 (2010).

3. Nigg, E. A. \& Raff, J. W. Centrioles, centrosomes, and cilia in health and disease. Cell 139, 663-678 (2009).

4. Schmidt, K. N. et al. Cep164 mediates vesicular docking to the mother centriole during early steps of ciliogenesis. J. Cell Biol. 199, 1083-1101 (2012).

5. Tanos, B. E. et al. Centriole distal appendages promote membrane docking, leading to cilia initiation. Genes Dev. 27, 163-168 (2013).

6. Lu, Q. et al. Early steps in primary cilium assembly require EHD1/ EHD3-dependent ciliary vesicle formation. Nat. Cell Biol. 17, 228-240 (2015).

7. Spektor, A., Tsang, W. Y., Khoo, D. \& Dynlacht, B. D. Cep97 and CP110 suppress a cilia assembly program. Cell 130, 678-690 (2007).

8. Pazour, G. J. et al. Chlamydomonas IFT88 and its mouse homologue, polycystic kidney disease gene tg737, are required for assembly of cilia and flagella. J. Cell Biol. 151, 709-718 (2000).

9. Qin, H., Diener, D. R., Geimer, S., Cole, D. G. \& Rosenbaum, J. L. Intraflagellar transport (IFT) cargo: IFT transports flagellar precursors to the tip and turnover products to the cell body. J. Cell Biol. 164, 255-266 (2004).

10. Pedersen, L. B. \& Rosenbaum, J. L. Intraflagellar transport (IFT) role in ciliary assembly, resorption and signalling. Curr. Top. Dev. Biol. 85, 23-61 (2008).

11. Garcia-Gonzalo, F. R. et al. A transition zone complex regulates mammalian ciliogenesis and ciliary membrane composition. Nat. Genet. 43, 776-784 (2011). 
12. Reiter, J. F., Blacque, O. E. \& Leroux, M. R. The base of the cilium: roles for transition fibres and the transition zone in ciliary formation, maintenance and compartmentalization. EMBO Rep. 13, 608-618 (2012).

13. Williams, C. L. et al. MKS and NPHP modules cooperate to establish basal body/transition zone membrane associations and ciliary gate function during ciliogenesis. J. Cell Biol. 192, 1023-1041 (2011).

14. Goetz, S. C., Liem, Jr K. F. \& Anderson, K. V. The spinocerebellar ataxiaassociated gene Tau tubulin kinase 2 controls the initiation of ciliogenesis. Cell 151, 847-858 (2012).

15. Cajanek, L. \& Nigg, E. A. Cep164 triggers ciliogenesis by recruiting Tau tubulin kinase 2 to the mother centriole. Proc. Natl Acad. Sci. USA 111, E2841-E2850 (2014).

16. Balla, T. Inositol-lipid binding motifs: signal integrators through protein-lipid and protein-protein interactions. J. Cell Sci. 118, 2093-2104 (2005).

17. Martin, T. F. Phosphoinositide lipids as signaling molecules: common themes for signal transduction, cytoskeletal regulation, and membrane trafficking. Annu. Rev. Cell Dev. Biol. 14, 231-264 (1998).

18. Niggli, V. Regulation of protein activities by phosphoinositide phosphates. Annu. Rev. Cell Dev. Biol. 21, 57-79 (2005).

19. Di Paolo, G. \& De Camilli, P. Phosphoinositides in cell regulation and membrane dynamics. Nature 443, 651-657 (2006)

20. Lemmon, M. A. Structural basis for high-affinity phosphoinositide binding by pleckstrin homology domains. Biochem. Soc. Trans. 27, 617-624 (1999).

21. Rebecchi, M. J. \& Scarlata, S. Pleckstrin homology domains: a common fold with diverse functions. Annu. Rev. Biophys. Biomol. Struct. 27, 503-528 (1998).

22. Lemmon, M. A. Membrane recognition by phospholipid-binding domains. Nat. Rev. 9, 99-111 (2008).

23. Jacoby, M. et al. INPP5E mutations cause primary cilium signaling defects, ciliary instability and ciliopathies in human and mouse. Nat. Genet. 41, 1027-1031 (2009).

24. Bielas, S. L. et al. Mutations in INPP5E, encoding inositol polyphosphate-5phosphatase E, link phosphatidyl inositol signaling to the ciliopathies. Nat. Genet. 41, 1032-1036 (2009).

25. Luo, N. et al. OCRL localizes to the primary cilium: a new role for cilia in Lowe syndrome. Hum. Mol. Genet. 21, 3333-3344 (2012).

26. Luo, N. et al. Compensatory role of inositol 5-phosphatase INPP5B to OCRL in primary cilia formation in oculocerebrorenal syndrome of Lowe. PloS One 8, e66727 (2013).

27. Wei, H. C. et al. Depletion of plasma membrane PtdIns(4,5)P2 reveals essential roles for phosphoinositides in flagellar biogenesis. J. Cell Sci. 121, 1076-1084 (2008).

28. Aukema, H. M., Chapkin, R. S., Tomobe, K., Takahashi, H. \& Holub, B. J. In vivo formation of polyphosphoinositide isomers and association with progression of murine polycystic kidney disease. Exp. Mol. Pathol. 57, 39-46 (1992).

29. Aukema, H. M., Yamaguchi, T., Takahashi, H., Celi, B. \& Holub, B. J. Abnormal lipid and fatty acid compositions of kidneys from mice with polycystic kidney disease. Lipids 27, 429-435 (1992).

30. Chavez, M. et al. Modulation of ciliary phosphoinositide content regulates trafficking and sonic hedgehog signaling output. Dev. Cell 34, 338-350 (2015).

31. Garcia-Gonzalo, F. R. et al. Phosphoinositides regulate ciliary protein trafficking to modulate hedgehog signaling. Dev. Cell 34, 400-409 (2015).

32. Xu, Q. et al. PIPKIgamma targets to the centrosome and restrains centriole duplication. J. Cell Sci. 127, 1293-1305 (2014).

33. Bielas, S. L. et al. Mutations in INPP5E, encoding inositol polyphosphate-5phosphatase E, link phosphatidyl inositol signaling to the ciliopathies. Nat. Genet. 41, 1032-1036 (2009).

34. Wei, Q. et al. Transition fibre protein FBF1 is required for the ciliary entry of assembled intraflagellar transport complexes. Nat. Commun. 4, 2750 (2013).

35. Ling, K. et al. Type I gamma phosphatidylinositol phosphate kinase modulates adherens junction and E-cadherin trafficking via a direct interaction with $\mathrm{mu}$ 1B adaptin. J. Cell Biol. 176, 343-353 (2007).

36. Ling, K., Schill, N. J., Wagoner, M. P., Sun, Y. \& Anderson, R. A. Movin' on up: the role of PtdIns $(4,5) \mathrm{P}(2)$ in cell migration. Trends Cell Biol. 16, 276-284 (2006).

37. Cajanek, L. \& Nigg, E. A. Cep164 triggers ciliogenesis by recruiting Tau tubulin kinase 2 to the mother centriole. Proc. Natl Acad. Sci. USA 111, E2841-E2850 (2014).

38. Gillingham, A. K. \& Munro, S. The PACT domain, a conserved centrosomal targeting motif in the coiled-coil proteins AKAP450 and pericentrin. EMBO Rep. 1, 524-529 (2000).

39. Hammond, G. R., Machner, M. P. \& Balla, T. A novel probe for phosphatidylinositol 4-phosphate reveals multiple pools beyond the Golgi. J. Cell Biol. 205, 113-126 (2014)

40. Balla, T. Imaging and manipulating phosphoinositides in living cells. J. Physiol. 582, 927-937 (2007).
41. Ferguson, C. G. et al. Phosphoinositide-containing polymerized liposomes: stable membrane-mimetic vesicles for protein-lipid binding analysis. Bioconjug Chem. 16, 1475-1483 (2005).

42. Odorizzi, G., Babst, M. \& Emr, S. D. Phosphoinositide signaling and the regulation of membrane trafficking in yeast. Trends Biochem. Sci. 25, 229-235 (2000).

43. Humbert, M. C. et al. ARL13B, PDE6D, and CEP164 form a functional network for INPP5E ciliary targeting. Proc. Natl Acad. Sci. USA 109, 19691-19696 (2012).

44. Narkis, G. et al. Lethal contractural syndrome type 3 (LCCS3) is caused by a mutation in PIP5K1C, which encodes PIPKI gamma of the phophatidylinsitol pathway. Am. J. Hum. Genet. 81, 530-539 (2007).

45. Wang, Y., Lian, L., Golden, J. A., Morrisey, E. E. \& Abrams, C. S. PIP5KI gamma is required for cardiovascular and neuronal development. Proc. Natl Acad. Sci. USA 104, 11748-11753 (2007).

46. Hsu, F., Hu, F. \& Mao, Y. Spatiotemporal control of phosphatidylinositol 4-phosphate by Sac2 regulates endocytic recycling. J. Cell Biol. 209, 97-110 (2015).

47. Levine, T. P. \& Munro, S. Targeting of Golgi-specific pleckstrin homology domains involves both PtdIns 4-kinase-dependent and -independent components. Curr. Biol. 12, 695-704 (2002).

48. Wang, Y. J. et al. Phosphatidylinositol 4 phosphate regulates targeting of clathrin adaptor AP-1 complexes to the Golgi. Cell 114, 299-310 (2003).

49. Follit, J. A. et al. The Golgin GMAP210/TRIP11 anchors IFT20 to the Golgi complex. PLoS Genet. 4, e1000315 (2008)

50. Lu, Q. et al. Early steps in primary cilium assembly require EHD1/EHD3dependent ciliary vesicle formation. Nat. Cell Biol. 17, 531 (2015).

51. Lev, S. Non-vesicular lipid transport by lipid-transfer proteins and beyond. Nat. Rev. 11, 739-750 (2010).

52. Gadi, D., Wagenknecht-Wiesner, A., Holowka, D. \& Baird, B. Sequestration of phosphoinositides by mutated MARCKS effector domain inhibits stimulated $\mathrm{Ca}(2+)$ mobilization and degranulation in mast cells. Mol. Biol. Cell 22, 4908-4917 (2011).

53. Corbalan-Garcia, S. \& Gomez-Fernandez, J. C. Signaling through C2 domains: more than one lipid target. Biochim. Biophys. Acta 1838, 1536-1547 (2014).

54. Jensen, V. L. et al. Formation of the transition zone by Mks5/Rpgrip1L establishes a ciliary zone of exclusion (CIZE) that compartmentalises ciliary signalling proteins and controls PIP2 ciliary abundance. EMBO J. 34, 2537-2556 (2015)

55. Ling, K., Doughman, R. L., Firestone, A. J., Bunce, M. W. \& Anderson, R. A. Type I gamma phosphatidylinositol phosphate kinase targets and regulates focal adhesions. Nature 420, 89-93 (2002).

56. Wang, Y. et al. Loss of PIP5KIgamma, unlike other PIP5KI isoforms, impairs the integrity of the membrane cytoskeleton in murine megakaryocytes. J. Clin. Invest. 118, 812-819 (2008).

57. Hammond, G. R., Schiavo, G. \& Irvine, R. F. Immunocytochemical techniques reveal multiple, distinct cellular pools of PtdIns4P and $\operatorname{PtdIns}(4,5) \mathrm{P}(2)$. Biochem. J. 422, 23-35 (2009).

58. $\mathrm{Xu}, \mathrm{Q}$. et al. BBS4 and BBS5 show functional redundancy in the BBSome to regulate the degradative sorting of ciliary sensory receptors. Sci. Rep. 5, 11855 (2015).

\section{Acknowledgements}

We thank Dr Marcelo Chávez (Université Libre de Bruxelles, Belgium), Drs Jeremy Reiter and Francesc Garcia-Gonzalo (UCSF, San Francisco, CA) for sharing their immunofluorescence staining methods. We thank Dr Kathryn Anderson (Sloan-Kettering Institute, New York, NY) for sharing the GFP-TTBK2 plasmid and Dr Seongjin Seo (University of Iowa, Iowa City, IA) for providing the Flag-INPP5E plasmid. We thank Trace Christensen and Scott Gamb (Electron Microscopy Core Facility, Mayo Clinic) for technical assistance with electron microscope studies. K.L., Q.X. and Y.H. are supported by research grants from NCI (1R01-CA149039) and NIDDK (DK90728). J.H., Y.Z. and Q.W. are supported by NIH/NIDDK (1R01-DK090038)

\section{Author contributions}

Q.X. contributed to study design, experiment execution, data analysis and manuscript editing. Y.Z. contributes to experiment execution and data analysis. Q.W. provided some reagents. Y.H. provided technical support. J.H. and K.L. supervised and contributed equally to study design and manuscript preparation, as well as providing project leadership. All authors discussed the results and commented on the manuscript.

\section{Additional information}

Supplementary Information accompanies this paper at http://www.nature.com/ naturecommunications

Competing financial interests: The authors declare no competing financial interests. 
Reprints and permission information is available online at http://npg.nature.com/ reprintsandpermissions/

How to cite this article: Xu, Q. et al. Phosphatidylinositol phosphate kinase PIPKI $\gamma$ and phosphatase INPP5E coordinate initiation of ciliogenesis. Nat. Commun. 7:10777 doi: $10.1038 /$ ncomms10777 (2016). (c) (i) This work is licensed under a Creative Commons Attribution 4.0 International License. The images or other third party material in this article are included in the article's Creative Commons license, unless indicated otherwise in the credit line; if the material is not included under the Creative Commons license, users will need to obtain permission from the license holder to reproduce the material. To view a copy of this license, visit http://creativecommons.org/licenses/by/4.0/ 